\title{
Restoring orbital thinking from real space descriptions: bonding in classical and non-classical transition metal carbonyls
}

\author{
Davide Tiana, $\dagger^{a b}$ E. Francisco,${ }^{c}$ M. A. Blanco, ${ }^{c}$ P. Macchi, ${ }^{b}$ Angelo Sironi ${ }^{a}$ and \\ A. Martín Pendás*c \\ Received 28th September 2010, Accepted 10th January 2011 \\ DOI: $10.1039 / \mathrm{c0cp01969k}$
}

A combined strategy that unifies our interacting quantum atoms approach (IQA), a chemically intuitive energetic perspective within the quantum theory of atoms in molecules (QTAIM), the domain natural orbitals obtained by the diagonalization of the charge-weighted domain-averaged Fermi hole (DAFH), and the statistical analyses of chemical bonding provided by the electron number distribution functions (EDF) is presented. As shown, it allows for recovering traditional orbital images from the orbital invariant descriptions of QTAIM. It does also provide bonding indices (like bond orders) and bond energetics, all in a per orbital basis, still invariant manner, using a single unified framework. The procedure is applied to show how the Dewar, Chatt, and Ducanson model of bonding in simple transition metal carbonyls may be recovered in the real space. The balance between the number of $\sigma$-donated and $\pi$-backdonated electrons is negative in classical compounds and positive in non-classical ones. The energetic strength of backdonation is, however, smaller than that of donation. Our technique surpasses conventional orbital models by providing physically sound, quantitative energetics of chemical bonds (or interactions) together with effective one-electron pictures, all for arbitrary wavefunctions.

\section{Introduction}

The vast majority of the concepts related to chemical bonding that we may find in a practicing chemist's toolkit stem from the orbital model. Orbitals are easy to parameterize, and orbital-based explanations have reached a high degree of predictivity over the years. ${ }^{1}$ However, any of such explanations is necessarily not invariant under general transformations that however preserve the wavefunction. This undesirable dependence is absent in real space theories of chemical bonding, which extract chemical information from invariant reduced density matrices. Many of them use the topology of a scalar field to partition the space into regions which are endowed with intuitive chemical meaning, for instance, the quantum atoms from the Quantum Theory of Atoms in Molecules (QTAIM), ${ }^{2}$ or the core, bonding, and lone pair basins obtained from the electron localization function (ELF) of Becke and Edgecombe. ${ }^{3}$

\footnotetext{
${ }^{a}$ Department of Structural Chemistry and Inorganic Stereochemistry, University of Milano, Italy

${ }^{b}$ Department of Chemistry and Biochemistry University of Bern, Switzerland

${ }^{c}$ Departamento de Química Física y Analítica, Facultad de Química, Universidad de Oviedo, 33006-Oviedo, Spain.

E-mail: angel@fluor.quimica.uniovi.es; Fax: + 34985 103125;

Tel: + 34985103037

$\dagger$ Previous address: Departamento de Química Física y Analítica, Facultad de Química, Universidad de Oviedo, 33006-Oviedo, Spain.
}

As a whole, these methods are becoming increasingly preferred over orbital based ones to analyze chemical bonding issues.

A salient feature of the QTAIM is that it provides not only a wealth of bonding indices by examining well defined scalars at a finite set of distinguished points (the critical points of the $\rho$ scalar field), but that it allows for exact partitions of the molecular energy. Bonding and binding (cohesion) may then be examined on the same footing. Our Interacting Quantum Atoms approach (IQA $)^{4-8}$ has been shown to provide a chemically appealing framework which unifies the standard QTAIM with a general theory of cohesion valid for any molecular geometry. In IQA, every pair of QTAIM quantum atoms interact among themselves, and on doing so their proper energies (self-energies) are altered. Binding appears as a competition between the promotion energy (deformation energy or self-energy change) of the atoms which is usually destabilizing, and their interatomic interaction energies, usually stabilizing. The interaction energies come from adding classical (or ionic-like), and quantum mechanical (or covalent-like) terms. IQA has been applied to shed light on a number of problems in chemical bonding, ${ }^{9-13}$ and we have recently analyzed the $\mathrm{M}-\mathrm{CO}$ link in both classical and non-classical carbonyls. ${ }^{14}$

However elegant these procedures may be, the penetration of real space reasonings in the chemical literature depends on the ability of the new methodologies to recast the orbital language into the new formalism without losing predictive 
power. This enterprise has not yet succeeded entirely, and the QTAIM, for instance, still lacks the predictivity of standard orbital-based models.

A possible way out of this situation is allowing the chemist to still use the deeply rooted orbital models within real space theories by endowing them with a one-electron operating mode. This may be done by reintroducing effective domainaveraged one-electron descriptions from purely invariant objects. These effective electrons may then be safely used to decompose our IQA atomic or interatomic quantities into orbital contributions, thus restoring orbital thinking from real space descriptions. In this work we develop such a strategy combining the IQA approach with the domain-averaged Fermi hole (DAFH) perspective introduced by Ponec. ${ }^{15,16}$ We have recently shown ${ }^{17}$ that a link exists between the DAFHs and the electron number distribution functions $(\text { EDFs })^{13,18-20}$ that provide the statistics of the electron population among different basins. This link shows that the DAFHs and their effective one electron functions are intimately linked to bonding concepts.

Our purpose in this paper is twofold. On the one hand, we will briefly present our IQA/DAFH/EDF combined strategy. On the other, we will apply it to show how to recover the Dewar, Chatt, and Duncanson model ${ }^{21,22}$ (DCD) of organometallic chemistry in simple carbonyls that is taken as a successful example of insights provided by orbital descriptions.

Our IQA image of the $\mathrm{M}-\mathrm{CO}$ bond has recently ${ }^{14}$ corroborated the general features of the DCD model, providing quantitative estimates of the ionic and covalent contributions to the metal-ligand link. However, orbital invariance precludes performing a direct decomposition of bond energetic quantities into $\sigma$-donation and $\pi$-backdonation from a pure real space technique. Thus, measures of the intensity of each of these contributions are to be indirect. For instance, by noticing that the covalent contribution to the metal-oxygen bond order, which is quantified in real space by its corresponding delocalization index, correlates with the classical notion of $\pi$-backdonation. ${ }^{23}$

All these limitations are surpassed by the new strategy, which we apply here to some of the complexes studied in our previous paper. ${ }^{14}$ We show how to quantify the $\sigma$ and $\pi$ contributions of the M-CO link, and how, as an interesting by-product of our analysis, a non-negligible multicenter character of $\pi$-backdonation appears as this contribution becomes larger.

We will first show the most relevant features of our combined strategy, focusing on the mutual relationships among quantities coming from different conceptual frames. Then we will briefly comment on computational details in Section 3. Section 4 will be devoted to present the effective orbitals for some chosen compounds, analyzing their contributions to both the $\mathrm{M}-\mathrm{CO}$ bond orders and covalent energies. Finally we will end up with some conclusions.

\section{The IQA/DAFH/EDF combined strategy}

We present the combined IQA/FH/EDF approach, which is here applied for the first time on prototypical compounds such as metal carbonyl complexes. Our real space description uses a QTAIM atomic partition of the space. IQA ${ }^{4-8}$ is then built upon it as an exact decomposition of the molecular energy into atomic and interatomic terms,

$$
E=\sum_{\mathrm{A}} E_{\mathrm{self}}^{\mathrm{A}}+\sum_{\mathrm{A}>\mathrm{B}}\left(V_{\mathrm{cl}}^{\mathrm{AB}}+V_{\mathrm{xc}}^{\mathrm{AB}}\right) \text {. }
$$

In this expression, $E_{\mathrm{self}}^{\mathrm{A}}$ is the self-energy associated to the quantum atom $\mathrm{A}$, constructed from kinetic and potential energy densities integrated over its own basin $\Omega_{\mathrm{A}}$, and $V_{\mathrm{cl}}^{\mathrm{AB}}$ and $V_{\mathrm{xc}}^{\mathrm{AB}}$ are the classical (ionic-like) and exchangecorrelation (covalent-like) interaction energies between atoms $\mathrm{A}$ and $\mathrm{B} . V_{\mathrm{cl}}^{\mathrm{AB}}$ gathers all the classical coulombic energy terms between the electron and nuclear densities in $\Omega_{\mathrm{A}}$ and $\Omega_{\mathrm{B}}$. $V_{\mathrm{xc}}^{\mathrm{AB}}$ provides the deviation of the $\mathrm{A}-\mathrm{B}$ electron-electron repulsion due to the quantum nature of the electron distribution. Since our concern in this work is showing how to recover orbital ideas from these covalent terms, we will mainly focus on $V_{\mathrm{xc}}^{\mathrm{AB}}$ :

$$
V_{\mathrm{xc}}^{\mathrm{AB}}=-\int_{\Omega_{\mathrm{A}}} \mathrm{d} \boldsymbol{r}_{1} \int_{\Omega_{\mathrm{B}}} \mathrm{d} \boldsymbol{r}_{2} \frac{\rho_{\mathrm{xc}}\left(\boldsymbol{r}_{1}, \boldsymbol{r}_{2}\right)}{r_{12}},
$$

where $\rho_{\text {xc }}\left(\boldsymbol{r}_{1}, \boldsymbol{r}_{2}\right)=\rho\left(\boldsymbol{r}_{1}\right) \rho\left(\boldsymbol{r}_{2}\right)-\rho_{2}\left(\boldsymbol{r}_{1}, \boldsymbol{r}_{2}\right)$ is the standard spinless exchange-correlation density. All the quantum properties of an electron system are contained in $\rho_{\mathrm{xc}}$, which may itself be averaged over a given basin to define the charge-weighted domain averaged Fermi (exchange-correlation, in general) hole (DAFH): ${ }^{15,16}$

$$
G^{\Omega}\left(\boldsymbol{r}_{2}\right)=\int_{\Omega} \rho_{\mathrm{xc}}\left(\boldsymbol{r}_{1}, \boldsymbol{r}_{2}\right) \mathrm{d} \boldsymbol{r}_{1},
$$

$G^{\Omega}$ provides a basin decomposition of the density, for $\rho(\boldsymbol{r})=$ $\sum_{i} G^{\Omega_{i}}(\boldsymbol{r})$, and its integrals over $\mathscr{R}^{3}, \Omega$, and $\Omega^{\prime} \neq \Omega$ recover important objects in the QTAIM: ${ }^{24,25}$

$$
\begin{aligned}
\int_{\mathscr{R}^{3}} G^{\Omega}(\boldsymbol{r}) \mathrm{d} \boldsymbol{r} & =N_{\Omega}, \\
\int_{\Omega} G^{\Omega}(\boldsymbol{r}) \mathrm{d} \boldsymbol{r} & =\lambda^{\Omega}, \\
\int_{\Omega^{\prime}} G^{\Omega}(\boldsymbol{r}) \mathrm{d} \boldsymbol{r} & =\delta^{\Omega, \Omega^{\prime}} / 2 .
\end{aligned}
$$

In the above expressions, $N_{\Omega}$ is the electron population of basin $\Omega, \lambda^{\Omega}$ its localization index, a measure of the number of electrons that do not delocalize to other regions of space, and $\delta^{\Omega, \Omega \prime}$ the delocalization index between basins $\Omega$ and $\Omega^{\prime}$, a standard measure of the covalent bond order in real space techniques. $^{26}$

Effective one-electron functions may be defined by diagonalizing the DAFH on the basis of occupied orbitals. ${ }^{15,16}$ In this way, $G^{\Omega}(\boldsymbol{r})=\sum_{i=1} n_{i}\left|\phi_{i}(\boldsymbol{r})\right|^{2}$, the $\phi_{i}$ 's being one-electron functions or domain natural orbitals (DNOs), and the $n$ 's a set of occupation numbers that reconstruct by summation $N_{\Omega}$. A nice link (exact for single determinant descriptions) between these DNOs, their occupation numbers, and the statistics of domain electron population exists. ${ }^{17}$ It establishes that the effective electrons described by the DNOs are statistically independent, so that $p\left(n_{\Omega}, n_{\Omega^{\prime}}\right)$, the probability of finding exactly $n_{\Omega}$ electrons in basin $\Omega$, and $n_{\Omega^{\prime}}=N-n_{\Omega}$ electrons in basin $\Omega^{\prime}=\mathscr{R}^{3}-\Omega$ is given by a binomial distribution constructed from a set of $N$ independent events (or coin tosses). Each of the electrons, described by one different $\phi_{i}$ has a probability $p_{i}(\Omega)=n_{i}=\int_{\Omega} \mathrm{d} \boldsymbol{r}_{1}\left|\phi_{i}\right|^{2}$ of being found in basin $\Omega$ (so that $\left.p_{i}\left(\Omega^{\prime}\right)=1-n_{i}\right)$. We should notice ${ }^{13}$ that 
the statistics of basin electron populations is intimately linked to electron localization and delocalization, and thus to chemical bonding. $^{24,25}$

The DNOs obtained for a given basin $\Omega$ come out in three basic flavors. Each $\phi_{i}$ may be either extremely localized in $\Omega$, with $n_{i} \approx 1$, extremely localized in $\Omega^{\prime}$, with $n_{i} \approx 0$, or partially delocalized between both, with an extreme case being $n_{i}=0.5$. DNOs are neither strictly localized orbitals (even though many of them are in fact localized in one basin), nor delocalized functions (even though some of them may be very delocalized). We prefer to use the term adaptive, for them, which reflects their more general nature more faithfully. It is easy to show that DNOs display the local symmetry of the $\Omega$ basin. In high symmetry situations this property is undesirable, and it is customary to break it by performing an isopycnic localization. ${ }^{27}$ This is a linear transformation, non-unitary, in general, that transforms the DNOs into a different set, the isopycnic orbitals, which fullfil three important properties: (i) they preserve the diagonal form of the one-particle density matrix, provided this is expressed in terms of the natural orbitals of the system; (ii) they transform according to the point group of the molecule, and (iii) they preserve the basic DNO properties (including their degree of localization) at the cost of mutual orthogonality that is rigorously lost. Nevertheless, this nonorthogonality is almost always residual in single determinant descriptions.

Rewriting eqn (2) and (4) in terms of DNOs lies at the core of our strategy. Let us start with eqn (4), which transforms easily into

$$
\begin{aligned}
N_{\Omega} & =\sum_{i} n_{i}, \\
\lambda^{\Omega} & =\sum_{i} n_{i}^{2}, \\
\delta^{\Omega, \Omega^{\prime}} & =2 \sum_{i} n_{i}\left(1-n_{i}\right),
\end{aligned}
$$

exact for single determinants. These three equations have a very simple interpretation. For instance, the average number of electrons in $\Omega$ is the sum of the probabilities of finding each electron in that basin. The last expression is particularly illuminating, since it decomposes the bond order as a sum of one electron contributions, just as done in naïve orbital approaches. And it does so in a particularly intuitive way: only electrons that delocalize between the basins contribute significantly to (covalent) bonding. The maximum contribution of a given electron to $\delta$ is 0.5 , this occurring when the electron is perfectly delocalized, $n_{i}=0.5$.

In this description, we may have either bonding delocalized orbitals, or non-bonding localized ones. The latter may be localized either in $\Omega$ or in $\Omega^{\prime}$. The decomposition of $\delta$ is also valid for any pair of basins at the single-determinant level, $\delta^{\mathrm{AB}}=2 \sum_{i} n_{i}^{\mathrm{A}} n_{i}^{\mathrm{B}}$. In the case of correlated descriptions the sums include more than $N$ terms, due to partially occupied natural orbitals, and are to be slightly transformed, but the general features here discussed remain. Results show that all core states give rise to extremely localized DAFH orbitals, and that bonding orbitals only appear from valence canonical functions, recovering textbook ideas. Mostly, any bonding orbital is only delocalized between two basins, but sometimes it covers non-negligibly the space of several. This allows for a simple real space mapping of multicenter bonding concepts, which have sometimes been said to lie beyond the QTAIM. $\delta$ 's are easy to generalize to what are called multicenter delocalization indices, ${ }^{28-33}$ which quantify the bond order associated to $n$-center links. These indices might also be used to define easy to compute quantities which correlate with back-bonding in more complex contexts. For instance, simple two-center metal-carbon delocalizations will fail to separate $\sigma$ and $\pi$ contributions in side on metal-olefin coordination complexes. We will pursue this issue in future publications.

Eqn (2) may also be rewritten in terms of DAFH orbitals. Considering a closed-shell single determinant for simplicity,

$$
\begin{aligned}
\rho_{\mathrm{xc}}\left(\boldsymbol{r}_{1}, \boldsymbol{r}_{2}\right) & =4 \sum_{i \geq j}^{N / 2} \phi_{i}\left(\boldsymbol{r}_{1}\right) \phi_{j}^{*}\left(\boldsymbol{r}_{1}\right) \phi_{i}^{*}\left(\boldsymbol{r}_{2}\right) \phi_{j}\left(\boldsymbol{r}_{2}\right), \\
V_{\mathrm{xc}}^{\Omega, \Omega^{\prime}} & =4 \sum_{i \geq j}^{N / 2} \int_{\Omega} \int_{\Omega^{\prime}} \mathrm{d} \boldsymbol{r}_{1} \mathrm{~d} \boldsymbol{r}_{2} \frac{\phi_{i}\left(\boldsymbol{r}_{1}\right) \phi_{j}^{*}\left(\boldsymbol{r}_{1}\right) \phi_{i}^{*}\left(\boldsymbol{r}_{2}\right) \phi_{j}\left(\boldsymbol{r}_{2}\right)}{r_{12}} .
\end{aligned}
$$

As we have already explained, ${ }^{17}$ the orthogonality of the DNOs in both $\Omega$ and $\Omega^{\prime}$ will make the non-diagonal $(i \neq j)$ contributions to $V_{\mathrm{xc}}^{\Omega \Omega^{\prime}}$ very small (strictly vanishing in the absence of the $r_{12}$ denominator). Moreover, the diagonal $i=j$ terms will be dominated, by large, by orbitals delocalized between $\Omega$ and $\Omega^{\prime}$. DNOs localized either in $\Omega$ or in $\Omega^{\prime}$ will contribute to one of the $\boldsymbol{r}_{1}$ or $\boldsymbol{r}_{2}$ domains, respectively, but not to the other. In the end, the total $V_{\mathrm{xc}}^{\Omega \Omega^{\prime}}$ energy will mainly come from diagonal delocalized, i.e. bonding, terms, and

$$
V_{\mathrm{xc}}^{\Omega \Omega^{\prime}} \approx \sum_{\phi_{i} \text { bonding }} V_{\mathrm{xc}}^{\Omega \Omega^{\prime}}(i i) .
$$

We have found this expression to recover about $90 \% V_{\mathrm{xc}}$ in standard cases, deviations coming basically from non-diagonal terms that couple different bonding orbitals. For instance, in $\mathrm{Li}_{2}-251.688$ out of $-251.709 \mathrm{~kJ} \mathrm{~mol}^{-1}$ of total $V_{\mathrm{xc}}^{\mathrm{LiLi}}$ is due to diagonal terms. In $\mathrm{N}_{2}$, with a much larger $V_{\mathrm{xc}}^{\mathrm{NN}}=$ $-2486 \mathrm{~kJ} \mathrm{~mol}^{-1}, 264 \mathrm{~kJ} \mathrm{~mol}^{-1}$ are due to non-diagonal contributions.

All these ideas provide a rather compact framework that expands considerably the applicability, predictability, and usefulness of real space bonding analyses for the practicing chemist. This is the first work in which such a strategy is presented jointly and applied to a chemically interesting case. Our combined strategy provides: (i) a QTAIM atomic partition, with its associated critical points, atomic interaction lines, and the enormous amount of wisdom accumulated from correlations among chemical concepts and properties at critical points; (ii) an exact (IQA) energetic decomposition, valid at any molecular geometry, that introduces intuitive chemical concepts like atomic promotion energies or ionic and covalent contributions to the interaction energy between every pair of atoms in the system; (iii) a set of statistically independent one electron functions (DAFH orbitals) which give additive contributions to bond orders between basins, and approximately additive terms to the above covalent energies, substantiating 
bond order-bond energy relationships; (iv) a chemically appealing picture of covalency in terms of delocalization of electrons.

\section{Selected examples and computational details}

One of the most attractive characteristics of chemical bonding in transition metal complexes is that paired electrons belonging to one of the two counterparts (the metal or the ligand) can be mutually donated. Thus, together with the donation of electron pairs from the ligand (similar to what occurs in Lewis acid-base complexes of main group elements) we have a back-donation from the metal to the ligand. The two mechanisms are not exclusive and usually they both concur to the bonding. One of the most well known donation/back-donation scheme is that proposed by Dewar, Chatt, and Duncanson (DCD) ${ }^{21,22}$ widely adopted to explain bonding in metal olefins ${ }^{34-37}$ or metal carbonyl complexes. ${ }^{38-45}$

As the $\mathrm{M}-\mathrm{CO}$ bond is regarded, and grossly speaking in standard orbital parlance, a $\sigma$-donation from the HOMO of $\mathrm{CO}$ into an empty orbital of $\mathrm{M}$ is accompanied by $\pi$-backdonation from a $\mathrm{d}$ orbital of $\mathrm{M}$ into the LUMO of CO. Such a simple model explains a large set of experimental facts, and even though it has been questioned many times, particularly after the discovery of non-classical carbonyls ${ }^{45-47}$ with $\mathrm{CO}$ stretching frequencies larger than those of free $\mathrm{CO}$, it has essentially survived up-to-date. The energetic role of $\sigma$ and $\pi$ contribution to bonding has also been examined, for instance through the energy decomposition analysis (EDA) technique. ${ }^{48}$ In our opinion, the root of some of these controversies lies in the absence of a single consistent, physically rooted method that provides, simultaneously, the bonding energetics and the orbital picture to interpret it. Our approach provides one.

In our previous paper on bonding in metal carbonyls, ${ }^{14}$ we selected a relatively wide set of systems described at the HF, DFT, and CASSCF levels that spanned both the classical and non-classical regimes and we analyzed them in terms of IQA. Succinctly, the ionic contributions to $\mathrm{M}-\mathrm{CO}$ (ML) bonding were shown to depend heavily on the coordination index of $\mathrm{M}$ and the formal charge of the complex, evolving from destabilizing to stabilizing on going from mono- towards hexa-coordination, and from positively to negatively charged molecules. Regarding covalency, $V_{\mathrm{xc}}^{\mathrm{ML}}$ decreases with the coordination number due to the saturation of the metal binding ability, but increases on injecting electrons in the system. As a result of the above balance, the total ML interaction, $E_{\mathrm{int}}^{\mathrm{ML}}$, is generally dominated by covalency. Using the correlation $^{23}$ between $\delta^{\mathrm{MO}}$ and the $\pi$-backdonation (extended to the energetic realm through $V_{\mathrm{xc}}^{\mathrm{MO}}$ ) the basic features of the DCD model were also corroborated. Modern insights, like the relationship between the $\mathrm{CO}$ distance and the covalency of the $\mathrm{CO}$ bond, ${ }^{49}$ or the fact that non-classical carbonyls may display non-negligible backdonation, were also recovered. Overall, our results are in agreement with standard orbital-based knowledge. ${ }^{47}$

We will choose a few of those systems here to exemplify how those results are translated into the DAFH/EDF language to provide a unified real space bonding picture. We will examine the tetrahedral $\left[\mathrm{Co}(\mathrm{CO})_{4}\right]^{-}, \mathrm{Ni}(\mathrm{CO})_{4}$, and $\left[\mathrm{Cu}(\mathrm{CO})_{4}\right]^{+} \mathrm{d}^{10} T_{\mathrm{d}}$ complexes, and the square planar non-classical $\left[\mathrm{Ni}(\mathrm{CO})_{4}\right]^{2+} \mathrm{d}^{8}$ molecule. In order to simplify our discussion, only $\mathrm{HF}$ results will be discussed. As shown in a previous work, ${ }^{14}$ correlation corrections do not alter the bonding landscape significantly. In fact, electron density topologies, IQA energetics, and EDFs were found to suffer only small changes upon inclusion of correlation. In contrast, the DAFH analyses do only have a simple, straightforward link to the other formalisms at the single determinant level. Inclusion of correlation effects in the present strategy requires a generalization which is not difficult, but cumbersome. This goal is currently being worked out and will be presented elsewhere. Our experience ${ }^{5,10}$ on generalizing IQA to correlated descriptions allows us to expect that its impact will also be small in this case.

All the computational details are inherited from the preceding paper. ${ }^{14}$ 6-31G(d,p) HF calculations were done with GAMESS, ${ }^{50}$ with Hay and Wadt small core relativistic effective core potentials (ECPs) used to simulate the transition metals. $^{51}$ IQA/DAFH/EDF analyses were done with our PROMOLDEN, ${ }^{52}$ and EDF $^{53}$ codes. PROMOLDEN integrations were performed using typically tight parameters, truncated at $l_{\max }=10$, so that interaction energies are significant to about $4-5 \mathrm{~kJ} \mathrm{~mol}^{-1}$. We have previously shown ${ }^{54}$ how small core ECPs may be safely used in QTAIM/IQA studies by using interatomic surfaces obtained through core reconstruction.

\section{Results and discussion}

We will examine in this section our results. We will first present in detail the $\mathrm{Ni}(\mathrm{CO})_{4}$ tetracarbonyl, a balanced system very well studied ${ }^{55,56}$ in which more or less half the stabilization is due to $\sigma$-donation, and the other half to $\pi$-backdonation. Then we will switch to show how that landscape evolves on changing the metal in the $\mathrm{d}^{10}$ systems. After this we will again discuss the square planar $\left[\mathrm{Ni}(\mathrm{CO})_{4}\right]^{+2}$ system in some detail, and show the clear bonding differences found in this nonclassical compound. Since the decomposition of covalent energies into DNO contributions is extremely CPU-intensive, we have decided not to explore here the rest of the systems that may be found in our previous contribution. ${ }^{14}$ Recent DAFH analyses of binuclear carbonyls have been published, ${ }^{57,58}$ although their spirit is quite different to that of this work.

\subsection{The $\mathrm{Ni}(\mathrm{CO})_{4}$ complex}

Let us first consider the DNOs obtained by: (i) diagonalizing the charge-weighted Fermi hole averaged over the metal basin; (ii) subjecting the resulting orbitals to an isopycnic localization (vide supra).

Since we are interested in ML bonding, we will only analyze those DNOs with occupation numbers, $n_{i}$, significantly different from zero, i.e. DNOs totally or partially localized on $\mathrm{M}$. There are 13 of them in our three tetrahedral compounds. Fig. 1 shows them for the $\mathrm{Ni}(\mathrm{CO})_{4}$ case. The first four displaying $n_{i}>0.999$ are almost entirely localized in the metal basin, and may intuitively be associated to the $3 \mathrm{~s}$ and $3 \mathrm{p}$ valence orbitals of the $\mathrm{Ni}$ atoms (let us not forget that the [Ar] 

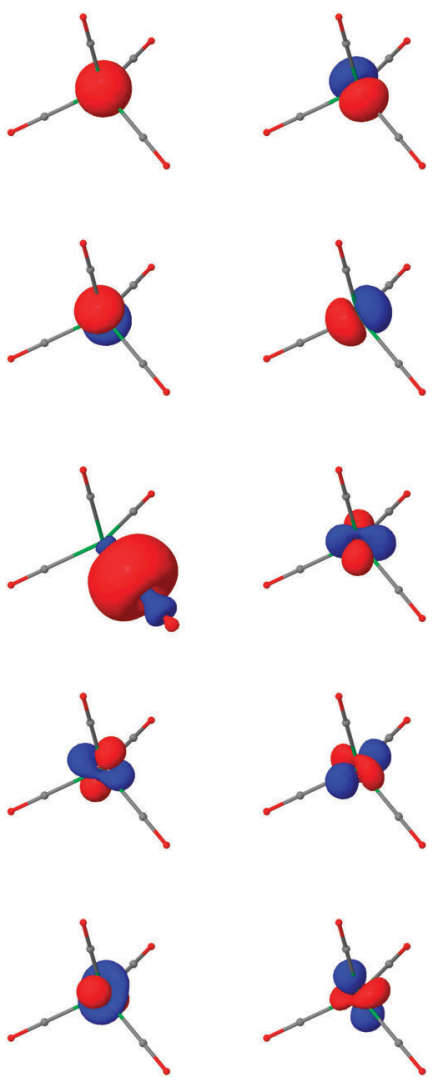

Fig. 1 Isopycnic DNO orbitals that are significantly localized on the $\mathrm{Ni}$ basin for the $\mathrm{Ni}(\mathrm{CO})_{4}$ tetracarbonyl. Isosurfaces displayed at the $|\phi|=0.05$ a.u. level. Only one of the four equivalent $\sigma$-like bonding orbitals is displayed. The order is from top to bottom, left to right, $\phi_{3 \mathrm{~s}}$, $3 \times \phi_{3 p}, \phi_{\sigma}, 5 \times \phi_{\mathrm{d}}$.

core has been substituted by an ECP). They do not participate in bonding.

The remaining nine functions are also immediately classified. Four of them $\left(\phi_{\sigma}^{i}, i=1, \ldots, 4\right.$, only one shown in the figure) are $\sigma$ orbitals delocalized over the metal and each of the four ligands, with an eigenvalue $n=0.117$. According to our EDF interpretation, they describe effective electrons that contribute to the ML bond. Notice that these bonding orbitals are rather polarized. If we obtain the domain integrals of $\phi^{2}$, the probability of finding the electron in the $\mathrm{M}$ basin turns out to be equal to 0.128 , and 0.838 in the corresponding $\mathrm{CO}$, so they are adequately interpreted as $\sigma$-donating carbonyl orbitals. Only 0.033 electrons described by $\phi_{\sigma}$ are delocalized among the other three remaining ligands. Another rationalization path would say that each ligand's $\phi_{\sigma}$ donates $0.117 \times 2=$ 0.234 electrons to the metal, making a grand total of about 0.935 electrons (see Table 1). The total delocalization index (bond order) between $\mathrm{M}$ and $\mathrm{L}$ is found to be $\delta^{\mathrm{ML}}=0.895$. With our decomposition, the contribution to this value coming from $\phi_{\sigma}$ is 0.407 ( 0.364 from the ligand's C, 0.027 from the ligand's $\mathrm{O}$, and 0.015 from the residual delocalization among the other three ligands). This value is only slightly smaller than half the total $\delta^{\mathrm{ML}}$. The rest up to 0.895 is due to the contribution of the five remaining DNOs, which, as seen in the figure are d-like functions very localized in the $\mathrm{Ni}$ basin,
Table 1 IQA/DAFH/EDF parameters for $\left[\mathrm{Co}(\mathrm{CO})_{4}\right]^{-}, \mathrm{Ni}(\mathrm{CO})_{4}$, and $\left[\mathrm{Cu}(\mathrm{CO})_{4}\right]^{+} T_{\mathrm{d}}$ tetracarbonyls. HF data in atomic units, except distances in $\mathrm{A}$, and frequencies in $\mathrm{cm}^{-1} . \Delta d(\mathrm{CO})$ and $\Delta \nu$ are changes with respect to the isolated ligand. $n$ 's are DNO occupancies, $N_{\mathrm{d}}$ is the total number of electrons donated via $\sigma$-donation channels to the metal, and $N_{\mathrm{b}}$ the total number of electrons backdonated via $\pi$ functions. Since each $\phi_{\sigma}$ is well-localized between the metal and one ligand, $\delta^{\mathrm{ML}}\left(\phi_{\sigma}\right)$, and $V_{\mathrm{xc}}^{\mathrm{ML}}\left(\phi_{\sigma}, \phi_{\sigma}\right)$ contain the contribution of only one $\sigma$ function. Delocalization of the other $\phi_{\sigma}$ 's over the chosen $\mathrm{L}$ is thus not summed up in the table. However, contributions of the five $\mathrm{d}$ functions to $\delta$ 's and $V_{\mathrm{xc}}$ 's have been averaged

\begin{tabular}{lccc}
\hline $\mathrm{M}$ & $\mathrm{Co}$ & $\mathrm{Ni}$ & $\mathrm{Cu}$ \\
\hline$d(\mathrm{MC})$ & 1.766 & 1.924 & 2.296 \\
$\Delta d(\mathrm{CO})$ & 0.024 & 0.002 & -0.010 \\
$\Delta \nu$ & -295 & -64 & 90 \\
$Q^{\mathrm{M}}$ & 0.189 & 0.122 & 0.802 \\
$n_{\sigma}$ & 0.131 & 0.117 & 0.056 \\
$n_{\mathrm{d}}\left(T_{2}\right.$-like $)$ & 0.729 & 0.892 & 0.974 \\
$n_{\mathrm{d}}(E-$-like $)$ & 0.848 & 0.894 & 0.975 \\
$N_{\mathrm{d}}$ & 1.047 & 0.935 & 0.448 \\
$N_{\mathrm{b}}$ & 2.235 & 1.058 & 0.254 \\
$\delta^{\mathrm{ML}}$ & 1.319 & 0.895 & 0.337 \\
$\delta^{\mathrm{MC}}$ & 1.153 & 0.798 & 0.313 \\
$\delta^{\mathrm{ML}}\left(\phi_{\sigma}\right)$ & 0.423 & 0.407 & 0.196 \\
$\delta^{\mathrm{ML}}\left(\phi_{\mathrm{d}}\right)$ & 0.850 & 0.477 & 0.124 \\
$V_{\mathrm{xc}}^{\mathrm{ML}}$ & -0.320 & -0.197 & -0.067 \\
$V_{\mathrm{xc}}^{\mathrm{MC}}$ & -0.302 & -0.187 & -0.065 \\
$V_{\mathrm{xc}}^{\mathrm{ML}}\left(\phi_{\sigma}, \phi_{\sigma}\right)$ & -0.139 & -0.096 & -0.042 \\
$V_{\mathrm{xc}}^{\mathrm{ML}}\left(\phi_{\mathrm{d}}, \phi_{\mathrm{d}}\right)$ & -0.129 & -0.075 & -0.020 \\
$V_{\mathrm{xc}}^{\mathrm{ML}}\left(\phi_{\sigma}, \phi_{\mathrm{d}}\right)$ & -0.033 & -0.026 & -0.005 \\
\hline
\end{tabular}

i.e. $\pi$-backdonating orbitals. We would like to stress how the adaptive localizability of the isopycnic DNOs provides a frame which recovers the DCD ideas straightforwardly.

A first point regarding these backdonating functions is that they do not localize over one particular CO moiety, contrarily to what the four $\sigma$ functions do. This points towards a very important difference between $\sigma$-donation and $\pi$-backdonation in these tetracarbonyls. In the former case, four isolated $\mathrm{ML}_{i}$ functions linking two-groups exist, while in the latter ML bonding must involve several ligands at a time, i.e. $\pi$ contributions are multiligand in nature. The five $\mathrm{d}$ DNOs come out to be slightly split in a $3+2$ fashion with $n$ 's equal to 0.892 and 0.894 , respectively. This splitting reminds the $T_{2}+E$ decomposition in $T_{\mathrm{d}}$ symmetry. Notice, however, that isopycnic orbitals do not rigorously transform as irreducible representations of the local point group, so that this resemblance should not be taken too far. Actually (see below), none of the five slightly split DNOs bind equally the four CO ligands, so that care is to be taken when labelled according to $T_{\mathrm{d}}$ irreducible representations. Each of the five $\mathrm{d}$ functions backdonates 0.213 electrons to each of the ligands, making a total of 1.058 electrons. The topological charge of the metal in the complex, +0.122 , may be viewed as coming from the balance of a $\mathrm{d}^{10}$ system backdonating 0.122 more electrons than those received from $\sigma$-donation from the ligands. In any case, five very localized d-like functions exist upon the metal, so our procedure allows us to assign a clear metallic configuration to this system.

On average, each of the $\mathrm{d}$ functions provides a total bond order with the rest of the system of 0.381 , of which 0.317 (about $85 \%$ ) is due to bonding with only two out of the four carbonyls. Each d function thus links preferentially the metal 


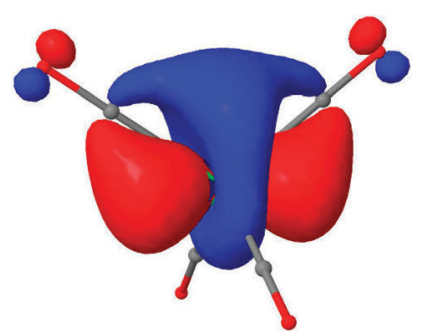

Fig. 2 Representative d isopycnic DNO orbital for the $\mathrm{Ni}(\mathrm{CO})_{4}$ tetracarbonyl. The isosurface has been expanded up to the $|\phi|=$ 0.03 a.u. level. Notice how the orbital is a combination of metal $\mathrm{d}$ and $\pi^{*}$ carbonyl functions, and that it preferentially links only two of the ligands.

to two L's, as seen in Fig. 2, where the expanded $|\phi|=0.03$ a.u. isosurfaces of one representative $\phi_{\mathrm{d}}$ function have been plotted. Any $\phi_{\mathrm{d}}$ may be described, roughly speaking, as an in-phase combination of one metal $\mathrm{d}$ and two $\pi^{*}$ orbitals from two ligands, again in agreement with the DCD picture. However, since 5 (d functions) is not congruent to 4 (carbonyls), the procedure freezes one out of 6 several equivalent resonance structures: since there are six different ligand pairs, and considering all five DNOs equivalent among themselves, we get one ligand pair not bonded by any DNO. This pair may be selected in six ways. We have thus a considerable multicenter character in backdonation. Adding the contribution of all the $\mathrm{d}$ functions to a given ML bond order we get a value of $0.381 \times 5 / 4=0.477$. Out of this value, $87 \%$ is due to the MC contribution, so only $13 \%$ may be assigned to the metal-oxygen delocalization, which has been used as a simple measure of backdonation. ${ }^{23}$ This $13 \%$ is significantly larger than the equivalent $7 \%$ in the $\sigma$ contribution, so the oxygen's share in $\pi$-backdonation is larger than in the case of $\sigma$-donation. Taken together with the $\sigma$ functions, $99 \%$ of the $\delta^{\mathrm{ML}}$ value is accounted for by the DCD donating and backdonating contributions.

A quantitative measure of the $\mathrm{M} \rightarrow \mathrm{LL}^{\prime}$ multicenter character of backdonation may be obtained from the so-called multicenter delocalization indices. ${ }^{28}$ Several definitions differing in normalization are available, so we will just provide the triple product of probabilities of finding an electron of a backdonating $\phi_{\mathrm{d}}$ in the $\mathrm{LL}^{\prime} \mathrm{M}$ triplet, $p(\mathrm{~L}) p\left(\mathrm{~L}^{\prime}\right) p(\mathrm{M})$. In Fig. 2, the product of the probabilities of finding an electron described by the depicted function in the metal center, and each of the two ligands at the top left and top right. From Table $1 p(\mathrm{~L})=p\left(\mathrm{~L}^{\prime}\right) \approx 0.05$, and $p(\mathrm{M}) \approx 0.89$ so the triple product is about 0.002 , to be compared with $1 / 27 \approx 0.037$, the equivalent three-center index in the ideal $3 c-2 e$ link in $\mathrm{H}_{3}^{+}$. Given the very polarized nature of these $\phi_{\mathrm{d}}$ 's, this is not such a small three-center index.

Let us now turn to the energetic realm. The covalent interaction energy associated to each $\mathrm{ML}$ link is $V_{\mathrm{xc}}^{\mathrm{ML}}=$ -0.197 a.u. $\left(-515 \mathrm{~kJ} \mathrm{~mol}^{-1}\right)$. Only $5 \%$ of this value is due to the MO interaction, as opposed to a larger $10 \%$ contribution in $\delta^{\mathrm{ML}}$. A decomposition into our DNO's works as expected. $87 \%$ of $V_{\mathrm{xc}}^{\mathrm{ML}}$ is due to the six diagonal contributions $V_{\mathrm{xc}}^{\mathrm{ML}}(i i)$ where $i$ runs over the appropriate $\phi_{\sigma}$, and the five $\phi_{\mathrm{d}}$ functions. If non-diagonal interactions between this set of six functions are included, this percentage grows to $95 \%$. This means that not only ML bond orders, but also covalent energies, are recovered from the set of appropriate $\sigma$ and $\pi$ contributions.

Out of the $-452 \mathrm{~kJ} \mathrm{~mol}^{-1}$ due to $V_{\mathrm{xc}}$ (ii) diagonal terms, -251 are solely due to $\phi_{\sigma}$, or $\sigma$-donation. The energetic share of each $\sigma$ bond is therefore similar, though slightly larger than its equivalent share in $\delta$. The remaining $-197 \mathrm{~kJ} \mathrm{~mol}^{-1}$ come from backdonation. Since, on average, $2.5 \phi_{\mathrm{d}}$ functions link the metal to each ligand, each of these links provides about $-80 \mathrm{~kJ} \mathrm{~mol}^{-1}$ to the covalent ML interaction energy. A second reading may focus on the $\phi_{\mathrm{d}}$ functions themselves, each of them backdonating to two ligands. In this view, every $\phi_{\mathrm{d}}$ accounts for $-159 \mathrm{~kJ} \mathrm{~mol}^{-1}$, about two thirds the $\sigma$ value. As the $V_{\mathrm{xc}}(i j)$ non-diagonal terms are concerned, we have found them almost exclusively due to couplings among the $\sigma-\mathrm{d}$ functions. $\sigma-\sigma$, and $\mathrm{d}-\mathrm{d}$ non-diagonal terms are very small, and no other relevant sources of non-diagonal contributions have been found. ML energetics, as well as ML delocalization is almost completely determined by the $\phi_{\sigma}, \phi_{\mathrm{d}}$ set of functions.

Summarizing, a $\sigma$-donating ML bond is about one and a half times stronger than any of the five three-center $\pi$-backdonations. Similarly, $\sigma$ delocalization is more effective than $\pi$ delocalization. The former accounts for $45 \%$ of the total $\mathrm{ML}$ $\delta$, a figure that is amplified to $55 \%$ as the covalent energy is regarded. Overall, our real space results are pretty compatible with MO arguments, ${ }^{56}$ but provide a far more detailed, invariant picture of bonding in this system.

\subsection{The DCD model in the $\mathrm{d}^{10} T_{\mathrm{d}}$ complexes}

After presenting a detailed picture in the Ni tetracarbonyl, let us now briefly turn to how all the above parameters change with $\mathrm{M}$. Table 1 gathers the more important results, including some overall IQA quantities already described in our previous paper. ${ }^{14}$ Since the IQA partitioning of the $V_{\mathrm{xc}}$ terms into orbital contributions is extremely CPU intensive, we have restricted our calculations to the cobalt and copper examples, with stronger and weaker ML bonding, respectively, than $\mathrm{Ni}(\mathrm{CO})_{4}$.

A first point regards the general nature of the DNOs. The same general structure found for the $\mathrm{Ni}$ case is repeated in all the systems. We have found extremely localized $3 \mathrm{~s}$ and $3 p$ functions that do not participate in bonding, plus a set of four equivalent localized $\phi_{\sigma}$ donating and five delocalized $\phi_{\mathrm{d}}$ backdonating orbitals. According to this view, all these systems may be described as $\mathrm{d}^{10}$ complexes. We should notice how the DNOs occupation numbers evolve on going from the copper to the cobalt complex: (i) $n_{\sigma}$ increases, so the $\sigma$-donating functions become more delocalized over the metal; (ii) $n_{\mathrm{d}}$ decreases, and the $\pi$-backdonating orbitals delocalize better over the ligands; (iii) the $T_{2}-E$-like splitting increases such that $n_{\mathrm{d}}\left(T_{2}\right)$ becomes smaller and the participation of the $T_{2}$-like functions in ML bonding more important, as we should intuitively expect from standard molecular orbital arguments related to ML overlap.

As in $\mathrm{Ni}(\mathrm{CO})_{4}$, the $\mathrm{d}$ functions delocalize over more than one ligand. The pattern found in $\left[\mathrm{Cu}(\mathrm{CO})_{4}\right]^{+}$is exactly the same as already presented in $\mathrm{Ni}(\mathrm{CO})_{4}$, but as the $T_{2}-E$-like gap increases, a shift is seen towards another pattern. 

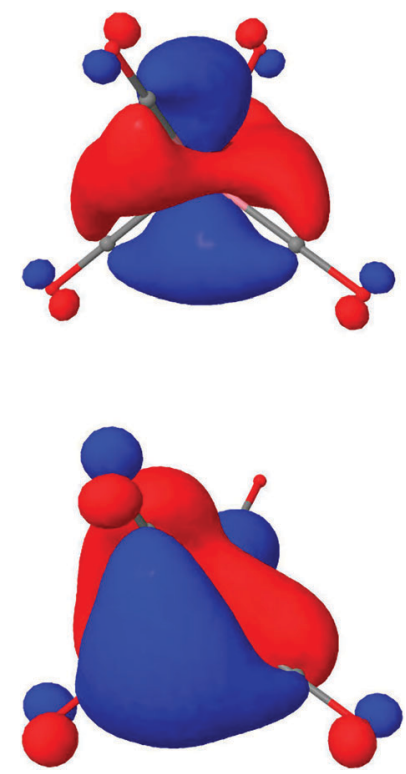

Fig. 3 Representative $\phi_{\mathrm{e}}$ (top), and $\phi_{t_{2}}$ (bottom) isopycnic DNO orbitals for the $\left[\mathrm{Co}(\mathrm{CO})_{4}\right]^{-}$complex. $|\phi|=0.05$ a.u. isosurfaces.

In $\left[\mathrm{Co}(\mathrm{CO})_{4}\right]^{-}$, the $e$-like functions link the four ligands at a time in a symmetric manner, while the $t_{2}$-like ones do only delocalize over three ligands, see Fig. 3. The multicenter (multiligand) character of backdonation thus increases as the formal charge of the complexes becomes more negative.

From the point of view of electron population, it is clear that both $\sigma$-donation and $\pi$-backdonation increase in the $\mathrm{Cu}$ to Co direction. This is clearly related to the decrease in $\mathrm{MC}$ distance. Overall, the topological charge on the metal may be seen as a balance between donation and backdonation over the ideal $\mathrm{d}^{10}$ configuration. As we shift from the copper towards the cobalt molecule, more electrons (thus stronger ML interaction) are delocalized both in the donating and backdonating channels. However, their mutual ratio changes completely. In the $\mathrm{Cu}$ case, the number of $\sigma$-donated $\left(N_{\mathrm{d}}\right)$ electrons almost doubles those $\pi$-backdonated $\left(N_{\mathrm{b}}\right)$. These numbers are roughly equal in the $\mathrm{Ni}$ molecule, but $N_{\mathrm{b}}$ more than doubles $N_{\mathrm{d}}$ in the Co complex. Thus, the positive topological charge of cobalt is the result of backdonating $2.235|e|$, but receiving only $1.047|e|$ from the ligands on top of its ideal -1 oxidation state.

Quantification of electron sharing (or electron delocalization) provides a similar picture, although backdonation (if not too large) is slightly more effective than donation to delocalize electrons. For that reason, the ratios of the $\pi$ to $\sigma$ contributions to $\delta^{\mathrm{ML}}$ are slightly larger than their equivalents obtained from the total number of electrons transferred, except in the $\left[\mathrm{Co}(\mathrm{CO})_{4}\right]^{-}$ case. In this latter system, the number of electrons shared via $\pi$-backdonation doubles those shared via $\sigma$ channels.

The energetic scale is again a product of these considerations, if we take into account that, as explained above, $\pi$ links do provide smaller bond energies than $\sigma$ ones. First, we notice that diagonal orbital terms account for about $90 \%$ of the total ML covalent energy. This fact corroborates the goodness of our approximations. Again, the only important non-diagonal terms providing contributions to $V_{\mathrm{xc}}$ in these systems are the

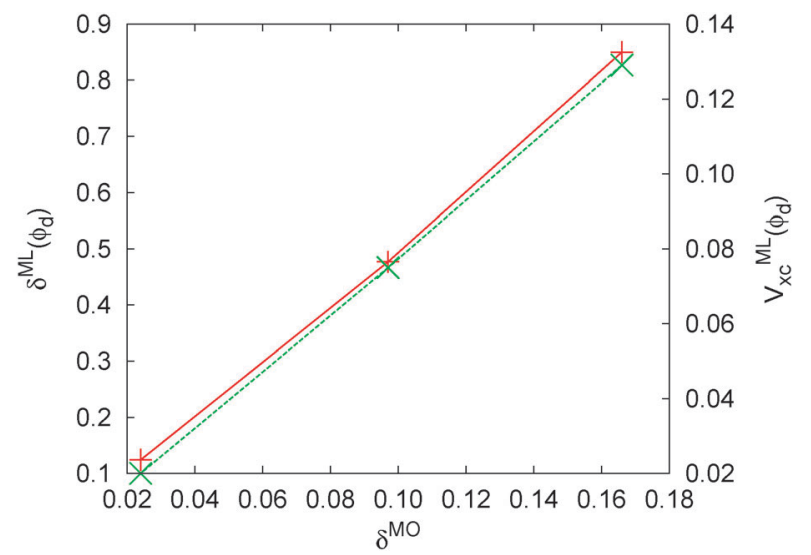

Fig. 4 Correlation between $\delta^{\mathrm{MO}}$ and both $\delta^{\mathrm{ML}}\left(\phi_{\mathrm{d}}\right)$ (solid line, left $y$ axis), and $V_{\mathrm{xc}}^{\mathrm{ML}}\left(\phi_{\mathrm{d}}\right)$ (dashed line, right $y$ axis) for the three $T_{\mathrm{d}}$ complexes. All data in a.u.

$\sigma-\pi$ ones, although in Co, very small other terms have also been found. Second, and contrarily to what it is found on examining charge transfers or electron delocalizations, the backdonation share in the ML covalent energy does not overcome the $\sigma$ contribution, although their ratio increases steadily from 0.5 to about 0.93 along the $\mathrm{Cu}$ to $\mathrm{Co}$ path. In the cobalt complex, each d function contributes, on average, about $-272 \mathrm{~kJ} \mathrm{~mol}^{-1}$ to the ML covalent energy.

An important point regards the use of simple correlations as measures of all these effects. Fig. 4 shows that the total $\delta^{\mathrm{MO}}$ value, as proposed by Macchi et al., ${ }^{23}$ may actually be taken as a reasonable measure of either $\delta^{\mathrm{ML}}\left(\phi_{\mathrm{d}}\right)$ or $V_{\mathrm{xc}}^{\mathrm{ML}}\left(\phi_{\mathrm{d}}\right)$, i.e. as an indirect measure of $\pi$-backdonation.

Finally, we note in passing that the change in $\mathrm{CO}$ distance and $\mathrm{CO}$ stretching frequency, basic parameters in the DCD model that we have successfully related in our previous work ${ }^{14}$ with the intensity of $V_{\mathrm{xc}}^{\mathrm{CO}}$, is connected in the present picture to the $N_{\mathrm{d}}-N_{\mathrm{b}}$ balance. This may again be quantified within our approach, but we will not pursue this point further in this work.

\subsection{The $\left[\mathrm{Ni}(\mathrm{CO})_{4}\right]^{2+}$ non-classical carbonyl}

Let us now discuss the clearly non-classical $\mathrm{d}^{8}$ square planar $\left[\mathrm{Ni}(\mathrm{CO})_{4}\right]^{2+}$ molecule. Our HF calculation shows a decrease in $\mathrm{d}(\mathrm{CO})$ of $0.02 \AA$, coupled to an increase in the CO stretching frequency of about $170 \mathrm{~cm}^{-1} \cdot{ }^{14}$ Only 12 isopycnic functions show non-negligible occupancies at the metal site. Fig. 5 shows them. Again, four of them, that may be made to correspond to the metal $3 \mathrm{~s}$ and $3 \mathrm{p}$ valence, display $n_{i}>0.998$ values, and do not contribute to bonding. We also recognize four equivalent $\sigma$-donating orbitals (only one shown), and four occupied d-like functions. The d space spans the $A_{1 \mathrm{~g}} \oplus B_{1 \mathrm{~g}} \oplus B_{2 \mathrm{~g}} \oplus$ $E_{\mathrm{g}}$ set of representations in $D_{4 \mathrm{~h}}$ symmetry, but the $B_{1 \mathrm{~g}}$-like representant is lacking from our DNOs. This indicates that the system may be described as a $\mathrm{d}^{8}$ one, again in agreement with formal electron counting. More interestingly, the unrepresented function, $\mathrm{d}_{x^{2}-y^{2}}$, is the only one having a zero overlap with a $\pi$ ligand contribution, reinforcing the intuitive role of the $\mathrm{d}$ functions as backbonding entities.

The four equivalent localized $\phi_{\sigma}$ 's display $n=0.086$, and are again very polarized. The probability that one of these 

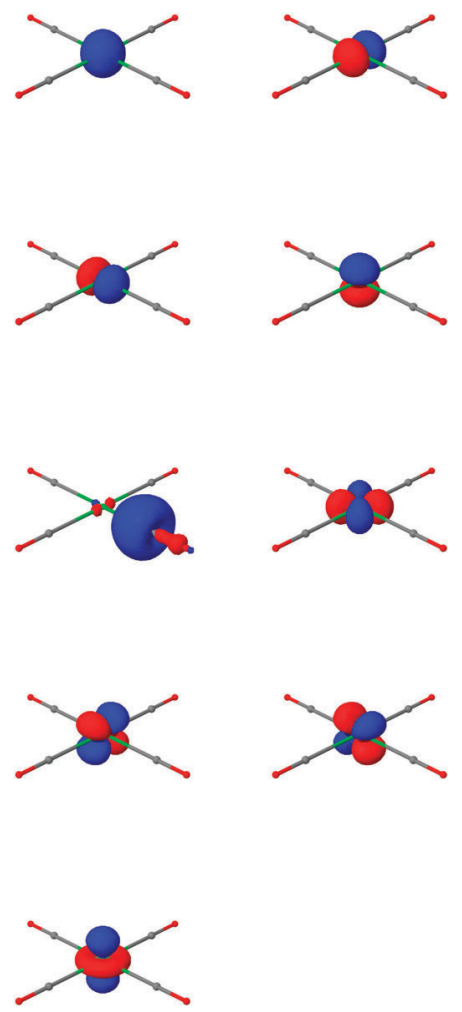

Fig. 5 DAFH isopycnic orbitals significantly localized on the $\mathrm{Ni}$ basin of the $\left[\mathrm{Ni}(\mathrm{CO})_{4}\right]^{2+}$ tetracarbonyl. Isosurfaces displayed at the $|\phi|=0.1$ a.u. level. Only one of the four equivalent $\sigma$-like bonding orbitals is shown. The order is from top to bottom, left to right, $\phi_{3 \mathrm{~s}}$, $3 \times \phi_{3 \mathrm{p}}, \phi_{\sigma}, 4 \times \phi_{\mathrm{d}}$.

effective electrons lies in the $M$ basin is equal to 0.100 , and 0.853 that it is found in its corresponding carbonyl, so about 0.047 electrons are found over other basins, basically in the trans carbonyl ligand ( 0.032 of them). As delocalization is concerned, $\delta^{\mathrm{ML}}=0.409$, and its contribution coming from $\phi_{\sigma}$ is $\delta^{\mathrm{ML}}\left(\phi_{\sigma}\right)=0.294$. Only $6 \%$ of this value is due to $\mathrm{MO}$ delocalization. The other three $\sigma$ functions contribute very little to the ML $\delta$, a mere total of 0.016 .

The $\mathrm{d}$ functions are very localized in the $\mathrm{M}$ basin, with $n$ 's equal to $0.976 \times 2,0.988$, and 0.969 in the $\mathrm{d}_{x z} \equiv \mathrm{d}_{y z}, \mathrm{~d}_{z^{2}}, \mathrm{~d}_{x y}$ order. They contribute to ML delocalization with $\delta^{\mathrm{ML}}\left(\phi_{\mathrm{d}}\right)=$ 0.107. Backdonation is therefore not large, but non-negligible. The four occupied d functions contribute differently to this value, the largest contribution being 0.041 for $\mathrm{d}_{x z}$ if $\mathrm{L}$ lies along the $x$ axis, for instance, and the smallest, 0.012 for $\mathrm{d}_{z^{2}}$, all in entire agreement with chemical intuition. We should notice that the $\mathrm{d}_{z^{2}}$-like function does not backdonate to $\pi$-like ligand functions, but to $\sigma$-like components.

Overall, 0.693 electrons are $\sigma$-donated to the metal, and only 0.184 backdonated to the ligands, making the total topological charge of the metal equal to 1.490 , the value reported in our earlier paper. Notice that in the copper tetrahedral compound $N_{\mathrm{b}}$ was about $55 \% N_{\mathrm{d}}$, but that here that figure has decreased to $26 \%$. The multicenter character of backdonation is again clear, the $\mathrm{d}_{z^{2}}$ and $\mathrm{d}_{x y}$-like DNOs backdonating equally to all the four ligands, while the $\mathrm{d}_{x z}, \mathrm{~d}_{y z}$ backdonate exclusively to two ligands trans to each other. Interestingly, $\sigma$-donation is also affected by this multicenter
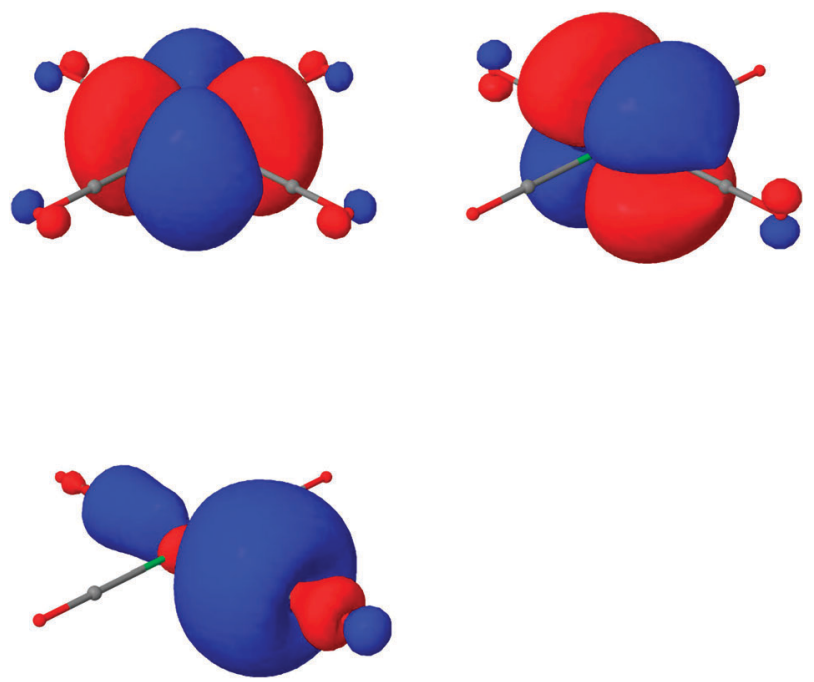

Fig. 6 Multicenter character of relevant DNOs in $\left[\mathrm{Ni}(\mathrm{CO})_{4}\right]^{2+}$. Isosurfaces displayed at the $|\phi|=0.007$, and 0.003 a.u. level for the $\mathrm{d}$ and $\sigma$ contributions, respectively.

delocalization, and each $\phi_{\sigma}$, as stated before, delocalizes slightly over its trans situated ligand. All these features are shown in Fig. 6. Notice that what is found here is the existence of direct delocalization channels that affect trans located ligands so that a perturbation at a given $\mathrm{L}$ will be transmitted directly to its trans partner. This effect may hold clues about the origin of the trans effect and deserves further exploration.

Finally, the total $V_{\mathrm{xc}}^{\mathrm{ML}}=-230 \mathrm{~kJ} \mathrm{~mol}^{-1}$ is partitioned in the following way: $-180 \mathrm{~kJ} \mathrm{~mol}^{-1}$ are due to $\phi_{\sigma}$, with only $-4 \mathrm{~kJ} \mathrm{~mol}^{-1}$ coming from the MO contribution, and $-42 \mathrm{~kJ} \mathrm{~mol}^{-1}$ are provided by the four $\phi_{\mathrm{d}}$ functions, so backdonation provides less than $20 \%$ of the total ML stabilization energy. Even more interesting is that $80 \%$ of the backdonation stabilization is due to two d functions: the $\mathrm{d}_{x y}$ and the appropriate $\mathrm{d}_{x z}$ or $\mathrm{d}_{y z}$ component. Finally, the remaining $8 \mathrm{~kJ} \mathrm{~mol}^{-1}$ needed to make the grand total of -230 are due to $\sigma-\mathrm{d}$ non-diagonal terms in $V_{\mathrm{xc}}$. So as far as covalency is regarded, backdonation is energetically small, but it clearly plays a role in bonding in these non-classical systems, in agreement with modern thinking.

\section{Conclusions}

We have presented in this paper a combined IQA/DAFH/ EDF strategy that allows us to recover effective one-electron functions from invariant descriptions coming from the Quantum Theory of Atoms in Molecules. Our Interacting Quantum Atoms approach (IQA) allows for a chemically appealing, exact real space decomposition of the total molecular energy at any geometrical configuration in terms of atomic self-energies and interatomic ionic and covalent interaction energies. Upon this invariant structure, the charge-weighted domain averaged Fermi hole natural orbitals originally defined by Ponec ${ }^{15,16}$ are obtained. We have recently shown them to be intimately linked to the statistics of the electron population (our electron distribution functions, $\mathrm{EDF}^{13,18}$ ) in real space basins. ${ }^{17}$ In fact, these effective electrons are statistically independent in one-determinant descriptions, and are adaptively localized or 
delocalized according to their non-bonding or bonding character. In the proposed strategy, only delocalized electrons contribute to bonding between two or more given QTAIM basins, and make an additive contribution to their mutual covalent bond order, as measured by the delocalization index, $\delta$. Since the strategy relies on a IQA energetic decomposition, the contribution of each of these electrons to the covalent energy may also be determined. In this way, a common framework that starts from the topology of the electron density to determine QTAIM atomic basins includes a detailed energetic view of bonding energies in terms of atomic deformations and electrostatic and covalent contributions, and provides an even thinner decomposition of the interaction energies in terms of one-electron functions is constructed.

We have applied this strategy to examine the DewarChatt-Ducanson model of bonding in simple transition metal carbonyls, and selected a subset of the systems previously analyzed at the IQA level. ${ }^{14}$ Our results show how we may easily recover $\sigma$-donation and $\pi$-backdonation from orbital invariant data, and quantify their role upon bonding, measured either as bond order (from delocalization indices) or bond strength (from IQA $V_{\mathrm{xc}}$ values). DNOs support the formal $d$ electron configurations traditionally assigned to the metals, and explain their globally positive topological charges in terms of the balance between the total number of donated and backdonated electrons. Even non-classical carbonyls display non-negligible backdonation, although the ratio of the strength of backbonding with respect to $\sigma$-donation is clearly smaller than one in these systems. In terms of the ML covalent energies, backdonation is weaker than $\sigma$-donation, and in none of the systems explored does the covalent energy coming from $\pi$ contributions exceed that emerging from $\sigma$ terms.

Another interesting point regards the multicenter character of ML bonding due to d-like contributions. It increases with the strength of backdonation, and is related to the well known trans effect in square planar compounds. Finally, our results confirm that $\delta^{\mathrm{MO}}$ is well correlated to the strength of backbonding, either measured by bond orders or bond energies, and that it may be used as an indirect index for it.

The strategy here developed shows how the chemical intuition developed through orbital arguments may indeed be used within real space theories of chemical bonding, moving us a step closer to obtaining chemically appealing one-electron images from general wavefunctions. At the time being, it offers unambiguous interatomic (or intergroup) interactions characterized by quantitative bond orders, with energies written as a sum of ionic and covalent contributions. The latter (and the bond orders) may be further decomposed into effective one-electron contributions which may be visualized in real space and used to develop standard orbital-like arguments. No other technique, to our knowledge, offers at the same time a quantitative energetic picture of bonding compatible with a one-electron (i.e. orbital) image.

\section{Acknowledgements}

D.T. thanks the University of Milan and the Italian Ministry of Research (MIUR) for a $\mathrm{PhD}$ grant that allowed his stay at Oviedo. EF and AMP acknowledge financial support from the
Spanish MICINN, Project No. CTQ2009-08376, the European Union FEDER funds, the MALTA-Consolider program (CSD2007-00045), and the FICYT (IB09-019). PM thanks the Swiss National Science Foundation for support (project 200021_125313). We devote this work to the memory of our friend, colleague, and author M. A. Blanco, who passed away during the preparation of this manuscript.

\section{References}

1 B. M. Gimarc, Molecular structure and bonding. The qualitative molecular orbital approach, Academic Press, New York, 1979.

2 R. F. W. Bader, Atoms in Molecules, Oxford University Press, Oxford, 1990.

3 A. D. Becke and K. E. Edgecombe, J. Chem. Phys., 1990, 92, 5397.

4 A. Martín Pendás, M. A. Blanco and E. Francisco, J. Chem. Phys., 2004, 120, 4581.

5 A. Martín Pendás, E. Francisco and M. A. Blanco, J. Comput. Chem., 2004, 26, 344.

6 M. A. Blanco, A. Martín Pendás and E. Francisco, J. Chem. Theory Comput., 2005, 1, 1096.

7 E. Francisco, A. Martín Pendás and M. A. Blanco, J. Chem. Theory Comput., 2006, 2, 90.

8 A. Martín Pendás, M. A. Blanco and E. Francisco, J. Comput. Chem., 2007, 28, 161.

9 A. Martín Pendás, E. Francisco and M. A. Blanco, J. Phys. Chem. A, 2006, 110, 12864.

10 A. Martín Pendás, M. A. Blanco and E. Francisco, J. Chem. Phys., 2006, 125, 184112.

11 A. Martín Pendás, M. A. Blanco and E. Francisco, J. Comput. Chem., 2009, 30, 98.

12 A. Martín Pendás, E. Francisco, M. A. Blanco and C. Gatti, Chem.-Eur. J., 2007, 13, 9362.

13 A. Martín Pendás, E. Francisco and M. A. Blanco, Phys. Chem. Chem. Phys., 2007, 9, 1087.

14 D. Tiana, E. Francisco, M. A. Blanco, P. Macchi, A. Sironi and A. Martín Pendás, J. Chem. Theory Comput., 2010, 6, 1064.

15 R. Ponec, J. Math. Chem., 1997, 21, 323.

16 R. Ponec, J. Math. Chem., 1998, 23, 85.

17 E. Francisco, A. Martín Pendás and M. A. Blanco, J. Chem. Phys., 2009, 131, 124125.

18 E. Francisco, A. Martín Pendás and M. A. Blanco, J. Chem. Phys., 2007, 126, 094102.

19 A. Martín Pendás, E. Francisco and M. A. Blanco, J. Phys. Chem. A, 2007, 111, 1084 .

20 A. Martín Pendás, E. Francisco and M. A. Blanco, J. Chem. Phys., 2007, 127, 144103.

21 M. Dewar, Bull. Soc. Chim. Fr., 1951, 18, C79.

22 J. Chatt and L. A. Duncanson, J. Chem. Soc., 1953, 2929.

23 P. Macchi, L. Garlaschelli and A. Sironi, J. Am. Chem. Soc., 2002, 124, 14173.

24 R. F. W. Bader and M. E. Stephens, J. Am. Chem. Soc., 1975, 97, 7391.

25 R. F. W. Bader and M. E. Stephens, Chem. Phys. Lett., 1974, 26, 445.

26 X. Fradera, M. A. Austen and R. F. W. Bader, J. Phys. Chem. A, 1999, 103, 304-314.

27 J. Cioslowski, Int. J. Quantum Chem., 1990, S24, 15.

28 R. C. Bochicchio, R. Ponec, A. Torre and L. Lain, Theor. Chem. Acc., 2001, 105, 292-298.

29 A. Torre, L. Lain and R. Bochicchio, J. Math. Chem., 2002, 32, 241.

30 R. Bochicchio, R. Ponec, L. Lain and A. Torre, J. Phys. Chem. A, 2000, 104, 9130 .

31 R. Bochicchio, R. Ponec, A. Torre and L. Lain, Theor. Chem. Acc., 2001, 105, 9130 .

32 M. Giambiagi, M. S. de Giambiagi and K. C. Mundim, Struct. Chem., 1990, 1, 423.

33 K. C. Mundim, M. Giambiagi and M. S. de Giambiagi, J. Phys. Chem., 1994, 98, 6118-6119.

34 U. Pidun and G. Frenking, Organometallics, 1995, 14, 5325.

35 R. H. Hertwog, W. Koch, D. Schröder, H. Schwarz, J. Hrusak and P. Schwerdtfeger, J. Phys. Chem., 1996, 110, 12253. 
36 G. Nicolas and F. Spiegelmann, J. Am. Chem. Soc., 1990, 112, 5410.

37 G. Frenking and N. Fröhlich, Chem. Rev., 2000, 100, 717.

38 T. Ziegler and A. Rauk, Inorg. Chem., 1979, 18, 1755.

39 F. Weinhold and C. Landis, Valency and Bonding. A Natural Bond Orbital Donor-Acceptor Perspective, Cambridge Univ. Press, 2005.

40 P. Macchi and A. Sironi, Coord. Chem. Rev., 2003, 238-239, 383.

41 F. Cortés-Guzmán and R. F. W. Bader, Coord. Chem. Rev., 2005, $105,3911$.

42 J. Pilme, B. Silvi and M. E. Alikhani, J. Phys. Chem. A, 2003, 107, 4506.

43 E. Matito and M. Solà, Coord. Chem. Rev., 2009, 253, 647.

44 A. J. Lupinetti, V. Jonas, W. Thiek, S. H. Strauss and G. Frenking, Chem.-Eur. J., 1999, 5, 2573.

45 P. K. Hurlbut, J. J. Rack, J. S. Luck, S. F. Dec, J. D. Webb, O. P. Anderson and S. H. Strauss, J. Am. Chem. Soc., 1994, 116, 10003.

46 A. J. Lupinetti, S. H. Strauss and G. Frenking, Prog. Inorg. Chem., 2001, 49, 1.

47 A. J. Lupinetti, G. Frenking and S. H. Strauss, Angew. Chem., Int. Ed., 1998, 37, 2113.
48 A. Diefenbach, M. F. Bickelhaupt and G. Frenking, J. Am. Chem. Soc., 2000, 122, 6449.

49 A. J. Lupinetti, S. Fau, F. Frenking and S. H. Strauss, J. Phys. Chem., 1997, 101, 9551.

50 M. W. Schmidt, K. K. Baldridge, J. A. Boatz, S. T. Elbert, M. S. Gordon, J. H. Jensen, S. Koseki, N. Matsunaga, K. A. Nguyen, S. J. Su, T. L. Windus, M. Dupuis and J. A. Montgomery, J. Comput. Chem., 1993, 14, 1347.

51 P. J. Hay and W. R. Wadt, J. Chem. Phys., 1985, 82, 299.

52 A. Martín Pendás, The PROMOLDEN code. Unpublished.

53 E. Francisco, A. Martín Pendás and M. A. Blanco, Comput. Phys. Commun., 2008, 178, 621.

54 D. Tiana, E. Francisco, M. A. Blanco and A. Martín Pendás, J. Phys. Chem. A, 2009, 113, 7963.

55 M. Doerr and G. Frenking, Z. Anorg. Allg. Chem., 2002, 628, 843.

56 A. W. Ehlers, S. Dapprich, S. F. Vyboishchikov and G. Frenking, Organometallics, 1996, 15, 117.

57 R. Ponec, G. Lendvay and J. Chaves, J. Comput. Chem., 2008, 29, 1387.

58 R. Ponec, G. Lendvay and M. R. Sundberg, J. Phys. Chem. A, 2008, 112, 9936. 